\title{
State regulation in adults scoring high versus low on ADHD symptomatology: a pupillometry study
}

\author{
Authors: Leonhard H. Drescher ${ }^{1}$, C. Nico Boehler ${ }^{2}$, \& Jan R. Wiersema ${ }^{1}$ \\ ${ }^{1}$ Department of Experimental Clinical and Health Psychology, Ghent University, Ghent, Belgium \\ ${ }^{2}$ Department of Experimental Psychology, Ghent University, Ghent, Belgium \\ Corresponding author: Leonhard H. Drescher, Department of Experimental Clinical and Health Psy- \\ chology, Ghent University, Henri Dunantlaan 2, 9000 Ghent, Belgium. \\ Email: leonhard.drescher@ugent.be
}

(C) 2021, American Psychological Association. This paper is not the copy of record and may not exactly replicate the final, authoritative version of the article. Please do not copy or cite without authors' permission. The final article will be available, upon publication, via its DOI:

$10.1037 /$ neu0000738 


\begin{abstract}
Objective: According to the state regulation deficit account, attention deficit/hyperactivity disorder (ADHD) is associated with difficulties in maintaining an optimal level of cognitive arousal. As the precise locus of this problem is yet unknown, the present study investigated this through behavioral and pupillometry indices. Method: Adults scoring high versus low on ADHD symptomatology carried out a target detection task at three event rate (ER) levels. Phasic pupil dilation was used as an index of cognitive effort, tonic pupil size as an index of tonic arousal. Results: Performance and self-reports indicated state regulation difficulties in the high-ADHD group. Phasic pupil dilation was increased during slow ER, indicating additional effort allocation. Surprisingly, tonic pupil size was smallest in the fast ER, and group effects were absent for both pupil measures. Conclusion: The high-ADHD group showed state regulation difficulties despite similar levels of additional effort allocation as reflected by phasic pupil responses.
\end{abstract}

Keywords: ADHD, state regulation, pupillometry 


\section{Key Points}

- The state regulation deficit (SRD) account of ADHD was tested in undiagnosed adults who scored high versus low on ADHD symptoms, using behavioral and pupillometry measures.

- Performance deficits and self-report differences were found in participants with higher levels of ADHD symptoms, indicating state regulation difficulties, yet no group differences were found in the pupil signal.

- While participants with higher levels of ADHD symptoms seemed to therefore invest the same amount of effort, this did not translate into equal performance.

- More research is called for to pinpoint the exact locus of ADHD-related state regulation difficulties. 


\section{Introduction}

Attention-deficit hyperactivity disorder (ADHD) is a neurodevelopmental disorder with symptoms of inattention and/or impulsiveness and hyperactivity, which has a worldwide prevalence of 5-7\% (American Psychiatric Association, 2013; Willcutt, 2012). It has its onset in childhood and persists until adolescence and adulthood in the majority of cases. ADHD impairs the functioning of the individual, causing social, academic, and occupational difficulties. Given the strong impact on the individual and its surroundings, the notably high prevalence, and the need for effective treatments, it is of importance to increase our understanding of the neurobiological mechanisms underlying ADHD-related behavior and symptom expression.

In the past decades, a growing body of research has provided support for the state regulation deficit (SRD) account of ADHD (Johnson et al., 2009; Metin et al., 2012; Sergeant, 2000; Sonuga-Barke et al., 2010; van der Meere, 2005). According to the SRD account ADHD-related symptoms and performance deficits are due to difficulties in regulating the cognitive arousal state in response to changing environmental conditions. This notion is based on studies that applied the Cognitive Energetic Model (CEM; Sanders, 1983) to ADHD. The resulting performance predictions of the CEM are closely related to the Yerkes-Dodson curve (Winton, 1987; Yerkes \& Dodson, 1908), which describes the relationship between physiological arousal levels and performance as an inverted U-shaped function. The CEM further postulates that an evaluation mechanism detects whether arousal levels are too low or too high, and triggers the allocation of top-down cognitive effort to up- or down-regulate arousal levels, when needed. In the CEM, a distinction is made between phasic and tonic aspects of arousal, referred to as 'arousal' and 'activation', respectively. Phasic arousal is defined as the short-lived, physiolog- 
ical response to input, while the tonic activation fluctuates over a longer time span and represents a general readiness for action (Pribram \& McGuinness, 1975). The SRD account is based on extensive research applying the CEM to ADHD and argues that ADHD symptoms and performance deficits emerge from sub-optimal activation (tonic arousal) levels, which may either be too low or too high for the concurrent task demands. There are several possible reasons for the deficient state regulation in ADHD: first, it could be due to an unstable activation system that is easily brought out of balance by changes in the context. Second, individuals with ADHD might not be able to compensate efficiently for non-optimal activation levels through the allocation of cognitive effort, either by not allocating the necessary effort or by an inefficient interaction between effort and activation mechanisms (Mohamed et al., 2016). In either way, on this account it follows that when the context (e.g., the task) is under- or overstimulating, both of which requiring effortful adjustments, people with ADHD are expected to perform worse than their peers. On the other hand, when the context provides an optimal level of stimulation, no performance differences are expected. In the absence of a manipulation of the arousal state, arousal-related measures such as visual processing speed may not be deficient in ADHD (Finke et al., 2011; Wiegand et al., 2016).

Numerous behavioral studies have provided results in support of the SRD account. This research covers a period of over three decades and was started in the 1980's by Sergeant and van der Meere (Sergeant, 2000; Sergeant \& Van der Meere, 1990). They applied the CEM to their ADHD research and systematically manipulated different parameters to investigate how the different energetic pools might be related to ADHD. There findings showed that the performance of individuals with ADHD was especially prone to manipulations of event rate (ER) (i.e. presentation rate of stimuli), which according to the CEM affects the activation level. Later studies consistently demonstrated a stronger impact of ER on performance in individuals with 
ADHD, suggesting difficulty in the regulation of the activation state (Börger \& van der Meere, 2000; Metin et al., 2014; Wiersema, Van der Meere, Antrop, et al., 2006; Wiersema, Van der Meere, Roeyers, et al., 2006). Most studies reported worse performance (slower reaction time; RT) in ADHD during slow ERs, suggesting a greater susceptibility to drift into a state of under-activation, which is not adequately counteracted by additional effort allocation. Psychophysiological research provided further evidence for the role of altered activation in state regulation difficulties in ADHD. Börger and van der Meere (2000) used heart rate deceleration as an index of motor activation and observed that individuals with ADHD showed decreased heart rate deceleration before responding, specifically during a slow event rate. Unfortunately, most of the aforementioned studies used only two ERs (fast and slow), which entails an obvious shortcoming, as this does not allow for an evaluation of quadratic effects. The SRD account predicts individuals with ADHD to show performance decrements in situations that elicit a non-optimal activation state due to difficulty regulating the activation state. Hence, this deficiency should be apparent when in an under-activated state but also when in an overactivated state. The few studies that used at least three ERs have indeed found ADHD-related differences in performance and brain functioning at the lower and upper end of the ER range (Benikos \& Johnstone, 2009; Metin et al., 2014; Metin, Krebs, Wiersema, Verguts, Gasthuys, van der Meere, et al., 2015; Scheres et al., 2001; van der Meere et al., 1995). In a meta-analysis by Metin et al. (2012), the authors found that ADHD and control group performance across experiments was in line with this inverted U-curve pattern. The results showed a disproportionate slowing of RT in ADHD relative to controls for slow ERs, while fast ERs led to inaccurate responding (viz., increased impulsive errors). Metin et al. (2014) used a task that included 4 ERs and showed that increased variability of responding in ADHD follows a U-curve shape. 
According to the CEM, additional effort allocation is required to regulate a non-optimal activation state. Event rate studies in ADHD that implemented psychophysiological (heart rate variability and event related potentials) and neuroimaging methods (functional Magnetic Resonance Imaging) indeed showed that individuals with ADHD allocated less effort specifically during non-optimal states (extreme event rates), while no difference was observed during more optimal event rates (Börger et al., 1999; Börger \& van der Meere, 2000; Metin, Krebs, Wiersema, Verguts, Gasthuys, Van der Meere, et al., 2015; Wiersema, Van der Meere, Roeyers, et al., 2006).

Despite the vast support for the SRD model in behavioral studies, effort allocation in ADHD is insufficiently tested with physiological markers. Therefore, we opted to measure pupil size in the current experiment, which has been argued to be the best autonomic index of cognitive effort allocation (Alnaes et al., 2014; Kahneman, 1973; Peavler, 1974). The amplitude of the phasic pupil reaction (i.e., the reaction of the pupil to task events) is correlated with task difficulty, which implies cognitive effort, and with subjective effort ratings across a wide variety of cognitive tasks (for a review, see van der Wel \& van Steenbergen, 2018). Evidence for this is drawn, for example, from conflict tasks where pupil reactions are larger on incompatible trials compared to compatible trials (van Steenbergen \& Guido, 2013).

One preliminary study applied pupillometry to investigate state regulation difficulties in ADHD (Metin et al., 2017). They found a U-shaped effect of ER on phasic pupil size in typically developing children, and a linear effect for the children with ADHD, indicating effort allocation difficulties in the fast ER for the ADHD group. However, this study had limited power due to a small sample size. Further, while the authors used phasic pupil dilation as an index of cognitive effort, tonic pupil size was not analyzed. 
The present study therefore also investigated tonic changes in pupil size (i.e., the baseline activity whose fluctuations are more sustained). According to the adaptive gain model of Aston-Jones and Cohen (2005), tonic pupil size reflects tonic arousal at the level of the Locus Coeruleus (LC) (Unsworth \& Robison, 2018; Wang et al., 2018), and resembles the concept of activation, the tonic aspect of arousal, as conceptualized in the CEM/SRD account.

The LC, located in the brainstem, is the main source of noradrenaline in the forebrain, and is critically involved in processes of arousal and attention (Hong, Walz, \& Sajda, 2014; Joshi, Li, Kalwani, \& Gold, 2016; Murphy, Robertson, Balsters, \& O’Connell, 2011; Rajkowski, Kubiak, \& Aston-Jones, 1994; Schneider et al., 2016; but see also Reimer et al., 2016). Aston-Jones and Cohen (2005) postulate that tonic LC activity relates to performance according to an inverted U-shaped function: performance is optimal when the tonic LC activation is in balance (i.e., moderate tonic activation). This $\mathrm{U}$-curve prediction resembles the $\mathrm{U}$-curve prediction of the CEM. Several researchers have argued that arousal regulation deficits in ADHD may be due to LC dysfunction (Howells et al., 2012; Imeraj et al., 2012; Konrad et al., 2006). This hypothesis, however, is yet to be confirmed.

Opting for pupillometry has implications for the choice of the experimental task. Phasic pupil reactions are rather slow and develop over the course of several seconds (Mathôt, 2018; Sirois \& Brisson, 2014). Most ER studies used a Go/No-Go task, containing frequent Go (response) trials and infrequent No-Go (inhibition) trials. However, in order to test the SRD account (Sonuga-Barke et al., 2010), it is not required for the task to tap into inhibition processes, as the Go/No-Go task does. Moreover, measuring pupil response during fast ERs in such a task is 
problematic due to overlapping responses to succeeding Go-trials. For this reason, we followed Metin et al. (2017) and opted for an oddball paradigm, more specifically a target detection task, which contains frequent non-response trials and infrequent response trials.

The current study set out to test hypotheses related to ADHD in a group design. An important consideration with respect to the sampling of participants for the current study is the recent consensus on the diagnosis of ADHD as the extreme end of a continuous distribution of ADHD symptoms in the population (Hudziak et al., 2007; McLennan, 2016). Therefore, we opted to recruit healthy participants without any diagnosed psychiatric condition, and to assess the ADHD symptom level by means of a questionnaire. This strategy has been used successfully in the past, and it has replicated ADHD-related differences in brain functioning and behavior (e.g., Broyd, Helps, \& Sonuga-Barke, 2011; Crosbie et al., 2013; Mohamed, Börger, Geuze, \& van der Meere, 2016; Nilsen, Mewhort Buist, Gillis, \& Fugelsang, 2013). Based on this questionnaire, we selected adults with particularly high and low scores and compared performance and pupil indices between them (i.e., high-ADHD and low-ADHD group).

Based on previous ADHD research (see the meta-analysis by Metin et al., 2012), we hypothesized a slowing of RT with decreasing ER, and that this effect would be stronger in the highADHD group, indicating state regulation difficulties. This would replicate patterns found in previous research by Metin et al. (2017) and other studies using Go/No-Go tasks with three ERs (Benikos \& Johnstone, 2009; Metin et al., 2014; Metin, Krebs, Wiersema, Verguts, Gasthuys, van der Meere, et al., 2015; van der Meere et al., 1995). While findings on RT variability are less consistent, based on the SRD account and Metin et al. (2014), we hypothesized adults with more ADHD symptoms to show increased variability at extreme ERs. We did not have clear hypotheses about accuracy (commission errors), as in contrast to previous Go/No- 
Go studies with a high inhibition load, not many errors were expected in the current used target detection task. With respect to the phasic pupil dilation, as an index of cognitive effort, we expected larger reactions during extreme ERs (over- and under-activated state), indicating compensatory cognitive effort required in these conditions. This would be reflected by a Ushaped effect across ERs. In the high-ADHD group, this U-shaped effect was predicted to be diminished, reflecting effort allocation difficulties. For the tonic pupil size, which we used as an index of activation (i.e., tonic arousal), we expected decreasing pupil size with deceasing ERs, representing a drop in activation levels. This effect was expected to be more pronounced in the high-ADHD group.

\section{Method}

This study was approved by the Ethics Committee of the Faculty of Psychology and Educational Sciences, Ghent University (approval no. 2018/83), and conducted in accordance with the Declaration of Helsinki. All participants provided written informed consent prior to the selection procedure and the experiment.

\subsection{Participants}

Participants were selected by means of a pre-screening questionnaire (see the procedure section for a detailed description). Out of 345 respondents, 158 received an invitation to participate in the experiment, based on their score. Seventy-six participants followed the invitation and completed the experiment (overall age range $18-29$ years), with 39 participants in the high-ADHD group (11 males, $M_{\text {age }}=20.9$ years, $S D_{\text {age }}=3.1$ years), and 37 participants in the 
low-ADHD group ( 8 males, $M_{\text {age }}=21.5$ years, $S D_{\text {age }}=2.9$ years). The groups were equal in gender ratio, $\mathrm{X}^{2}(1)=0.44, p=.51$, and age, $t(74)=0.87, p=.39$. All participants had normal or corrected-to-normal vision. They were remunerated with EUR 10 for their participation. For the analysis of the behavioral data, no participants were excluded, since the accuracy on the target detection task was excellent (>97\% for all participants). For the analysis of the pupil data, some datasets had to be removed because of missing data caused by technical failure of the eye tracking device. Since the number of excluded participants slightly varied dependent on the type of data to be analyzed, the final sample sizes are reported separately for each analysis in the results section.

\subsection{Selection procedure}

The experiment was advertised on social media platforms targeting university students, through flyers on campus, and on a faculty platform specifically designed for the recruitment of participants for psychological experiments. Inclusion was limited to student-age participants (18-30 years) without a formal diagnosis of any psychiatric or neurologic disorder, including ADHD, although one participant from the high-ADHD group reported a diagnosis of ADHD during the experiment. All potential participants were given the link to an online version of the short Adult ADHD Self-Report Scale, v1.1 (ASRS; Adler et al., 2006; Kessler et al., 2005). This questionnaire consists of 6 questions aimed at assessing key ADHD symptoms, each to be answered on a 5-point Likert scale. Often used as a short-form screener, it has been shown to have the highest predictive value for ADHD (Kessler et al., 2005, 2007). Before filling in the questionnaire, respondents were asked to read and accept an informed consent. Additionally, the importance of providing honest answers was stressed. While the ASRS is frequently scored 
in a categorical fashion to classify clinical ADHD, in the present study we scored the ASRS dimensionally by computing the sum score of each participant across the six questions (scores: $0=$ never, $1=$ rarely, 2 = sometimes, $3=$ often, $4=$ very often). This procedure has been recommended for repetitive use (Kessler et al., 2005), and was appropriate for our current design as we approached ADHD as a continuous trait. Participants with extreme scores on the ASRS were selected, and cut-offs were established based on the distribution of the pre-screening sample. The basis for this sample was a pre-existing collection of $n=50$ datasets. This subsample was obtained from an online prescreening platform of the faculty, where interested participants could complete a set of questionnaires, based on which they could be contacted for experiments. Additional questionnaire responses for the current experiment were collected online (via LimeSurvey) and added to the pre-existing set of potential participants. Invitations were sent out to eligible respondents, scoring either above or below the cut-offs of the pre-screening sample. Invitees could then voluntarily sign up for a time-slot on the recruitment platform, in order to take part in the full experiment. To obtain equal group sizes, the cut-offs were dynamically adapted according to response rates, although it was ensured that they remained above and below the interquartile range. The final cutoffs were a sum score of $\geq 13$ for the high-ADHD group and $\leq 6$ for the low-ADHD group (with one participant exceptionally scoring 7). This recruitment strategy resulted in gender- and age-balanced groups in terms of demographics (see above), whilst ensuring the largest possible difference in ADHD symptomatology in the given participant pool. Both post-hoc questionnaires confirmed a strong group difference in ADHD-symptom levels (see results section). Furthermore, when applying the categorical scoring method, 32 out of the 39 participants of the high-ADHD group obtained scores above the clinical threshold.

\subsection{Materials}


For this experiment, a two-computer setup was used. On the first computer, the experimental tasks were run using "Presentation" software (Version 18.0, Neurobehavioral Systems, Inc.). The pupil data were recorded using a Tobii TX300 eye tracking device (Tobii Technology AB, 2014) with a sampling frequency of $300 \mathrm{~Hz}$. This eye tracker allows for (limited) head movement, as the built-in sensors automatically detect the position of and distance to the eyes. It is furthermore equipped with a 23" monitor with a resolution of $1920 \times 1080$ and a screen response time of $5 \mathrm{~ms}$. The data generated by the eye tracker were recorded on the second computer using Tobii Studio software (Tobii AB, 2016). Tobii Studio received real-time event markers from the first computer through a plugin for Presentation (FH Johanneum, 2018) via an Ethernet connection. Behavioral reactions were recorded on the first computer.

Two post-hoc questionnaires were filled out by the participants. The first was the full ASRS, which comprises 18 questions, to be answered on a 5-point Likert scale (Adler et al., 2006; Kessler et al., 2005), including the ones from the short ASRS, which was used in the prescreening procedure. In the official ASRS publication of the World Health Organization, some of the answering boxes on each question are shaded to indicate the clinical cutoff on the question level (Kessler et al., 2005). Since this could create a bias for dimensional scoring, the shades were removed for this experiment (Panagiotidi et al., 2017). The second questionnaire (hereafter referred to as "SRD questionnaire") contained 10 questions about state regulation difficulties, also with a 5-point Likert response scale ranging from $0=$ "much less than average" to $4=$ "much more than average". Importantly, this questionnaire is designed to assess both over- and under-stimulation and the regulation thereof, while refraining from creating overlap with ADHD symptoms as defined in the ASRS or the DSM. An example item of this instrument is "I find it easy to calm down after an exciting situation". The SRD questionnaire was recently 
developed by our research team and can be found in Supplementary Materials 1 . For both the ASRS and the SRD, dimensional sum scores were used.

\subsection{Task and Stimuli}

The target detection paradigm consisted of two stimulus types: a frequent "No-Go" or standard stimulus (the letter "O", $70 \%$ of the trials), and an infrequent "Go" or target stimulus (the letter " $Q$ ", 30\% of the trials). Figure 1 provides a schematic representation of the task. The stimulus appeared on the screen between two vertical bars which were continuously present throughout the task. In this paradigm, the stimuli were the only intermittent source of stimulation (i.e., there was no visual overlap with fixation aid). The stimuli were presented for 300 ms, separated by an inter-stimulus interval (ISI) which was slightly jittered. Three ER conditions (fast, moderate and slow) were administered in a counterbalanced order. In the fast ER condition, the ISI was $1000 \mathrm{~ms}$ ( $\pm 100 \mathrm{~ms}$ jitter), in the moderate condition it was $4000 \mathrm{~ms}( \pm 300$ ms jitter), and in the slow condition it was $8000 \mathrm{~ms}$ ( $\pm 400 \mathrm{~ms}$ jitter). The letters had a vertical size of $5.1^{\circ}$ visual angle and a horizontal size of $3.8^{\circ}$, the two vertical bars $7.2^{\circ}$ (their horizontal distance being $10.3^{\circ}$ ). Both the stimuli and the bars were presented in dim red (RGB 170, 88, 88 ) against a grey background (RGB 108, 110, 108). These colors were chosen in order to be equiluminant ${ }^{1}$ and thus avoiding luminance-dependent pupil reactions. The order of the trials was pseudo-randomized with a pre-established sequence that was the same for all participants. To achieve a comparable duration of the ER conditions (Metin et al., 2014), the number

\footnotetext{
${ }^{1}$ For the calibration of the equiluminance of the stimulus material, a Konica Minolta LS-100 luminance meter was used (Konica Minolta, 2013). The RGB values were adjusted to reach a matching surface luminance of the stimuli and the background. This resulted in a constant luminance of $39.5 \mathrm{~cd} / \mathrm{m}^{2}$ for both the stimuli and the background in this specific setting.
} 
of trials was set to 320 for the fast ER, 100 for the moderate ER and 60 for the slow ER. In the beginning, middle and end of each ER condition, a 15-s baseline period was presented, consisting of the two vertical bars without presenting any stimulus. The rationale of this procedure was to obtain an additional measure of tonic pupil size, with reduced influences of stimulus presentation and motor activity (the other index being the pre-stimulus baseline pupil size). At the end of each ER version, this baseline period was followed by a few extra trials which continued for approximately $1 \mathrm{~min}$, in order to prevent participants from predicting the end of the condition after the last baseline period, and thus losing their task focus. Together, this resulted in a duration of approximately 10 min per condition.

[INSERT FIGURE 1]

\subsection{Experimental procedure}

Upon arrival, participants were verbally instructed by the experimenter about the course of the experiment and the use of the eye tracker. The seat and the eye tracking device were adjusted to their body size. Although movement was not restricted physically, participants were asked to remain at a constant distance of approximately $60 \mathrm{~cm}$ from the screen throughout the experiment. After a 9-point calibration procedure, participants completed ten practice trials with feedback about the accuracy of the response. Before the start of the main experiment, on-screen instructions reminded them about the importance of remaining still and looking at the screen. They were also instructed to respond both fast and accurately to the stimuli. The ER conditions were separated by breaks with a minimum duration of $60 \mathrm{~s}$. During these breaks, participants were asked to let their eyes rest, but remain seated in the same position. 
The eye tracker was recalibrated if the participant could not remain in the same position throughout the experiment.

\subsection{Analysis}

\subsubsection{Behavioral data}

For the analysis of the RT, all correct responses were included, except the first response of the condition and the first response after the mid-condition baseline period. Median values and the quartile-based variability coefficient (inter-quartile range/median) were obtained per participant and condition. We opted for the quantile-based approach to central tendency and variability, because a large portion of the individual RT distributions were non-normally distributed, as shown by Kolmogorov-Smirnov normality tests. The quantile-based approach solved this problem as it is robust against outliers and skewed distributions. As the distribution of the medians and variability coefficients on the group level in turn met the normality assumption (again shown by KS-tests), classical ANOVA was selected for the calculation of the results. Importantly, when using average RT as a central tendency parameter and the respective coefficient of variability $(M / S D)$ on the individual level, the group level results did not change substantially. Error rates could not be analyzed, since a substantial portion of the participants achieved an accuracy of $100 \%$. The statistical models were calculated in SPSS and JASP (JASP Team, 2020). For the ER $\times$ Group interactions, we report the polynomial contrasts, as we had specific predictions related to the nature of these interactions (linear, quadratic).

\subsubsection{Pupil data}


The following steps were used to process the pupil data. The raw eye tracker output was preprocessed in SPSS, removing unnecessary data and inserting markers for the behavioral responses. Once the data were pre-processed, they were loaded into BrainVision Analyzer 2 (BrainProducts) using a plugin created by van Steenbergen (2008). The pupil signal was averaged across both eyes, after which blinks (i.e., gaps of missing values) were linearly interpolated, again using code by van Steenbergen (2008). This interpolation was set to start $40 \mathrm{~ms}$ before the gap and end $40 \mathrm{~ms}$ thereafter (Zénon, 2017). The exact interval (40 ms) was selected based on the observation that it greatly removed the noise in the gap onset-locked and the gap offset-locked pupil signal. The signal was then segmented into trials, and baselinecorrected using the 200 ms pre-stimulus period. The trials were subsequently averaged within conditions and participants, and a $4 \mathrm{~Hz}$ low-pass-filter was applied to the average signal. Trial segments ranged from $-1500 \mathrm{~ms}$ to $+2500 \mathrm{~ms}$ in the fast condition (comprising three trials), $200 \mathrm{~ms}$ to $+4000 \mathrm{~ms}$ in the medium condition, and $-200 \mathrm{~ms}$ to $+8000 \mathrm{~ms}$ in the slow condition. For the analysis of the transient (phasic) pupil reaction, the baseline-corrected maximum of the pupil size following target trials was detected with a semi-automatic procedure which allowed for visual inspection and correction. The default search interval was $600-2500 \mathrm{~ms}$ post-stimulus onset, although in some cases the peak was manually defined outside of that interval. In order to test the validity of the point peak amplitude measure, the results were also calculated using an area of $100 \mathrm{~ms}$ around the peak. This did not have any significant impact on the results. Hence, point peak measures were used for the results.

In the fast ER condition, trials were analyzed in triplets, where the previous and subsequent trials were included. Given that the pupil reaction cannot fully unfold over a time span of merely $1300 \mathrm{~ms}$, this enabled the analysis of the full pupil reaction. For target trials, all valid 
triplets with the combination standard-target-standard were selected, while for standard trials, the combinations standard-standard-standard were used. The main features of the pupil reaction, i.e., a clear dilation following target trials and a clear constriction following standard trials, remained intact in the fast ER condition (see Figure 2).

\section{[INSERT FIGURE 2]}

Two distinct indices of tonic pupil size were used in the analysis. Firstly, the 200 ms pre-stimulus baseline segments were used as an index of tonic pupil size which takes into account its trial-by-trial fluctuations (Hong et al., 2014). Here, the average of all 200 ms pre-stimulus segments was obtained for each condition. Secondly, we analyzed the $15 \mathrm{~s}$ baseline period sampled halfway through the condition. This mid-condition baseline period is independent from possible startup or fatigue effects and therefore the most valid index of ER-induced activation level. Data from the baseline period were excluded from analysis if they contained less than 5 $\mathrm{s}$ of valid data. The average pupil dilation was obtained for the $15 \mathrm{~s}$ interval. For all pupil measures, the statistical analyses were carried out in JASP (JASP Team, 2020).

\section{Results}

\subsection{Questionnaires}

The average results of the full ASRS sum scores were $M_{\text {high }}=41.3, S D_{\text {high }}=7.6$ for the highADHD group and $M_{\text {low }}=17.8, S D_{\text {low }}=6.2$ for the low-ADHD group. The groups differed significantly, $t(74)=-14.60, p<.001$, Cohen's $d=3.36$, Bayes' Factor $B F>1000$. Additionally, when 
scoring the full ASRS in the categorical fashion instead of the sum score (see above), the average scores were $M_{\text {high }}=10.5, S D_{\text {high }}=3.1$ for the high-ADHD group, and $M_{\text {low }}=1.8, S D_{\text {low }}=1.9$ for the low-ADHD group, which confirms the strong difference in ADHD symptoms between both groups (Garnier-Dykstra et al., 2010).

The scores from the SRD questionnaire were $M_{\text {high }}=24.1, S D_{\text {high }}=4.0$ for the high-ADHD group and $M_{\text {low }}=18.3, S D_{\text {low }}=3.5$ for the low-ADHD group. Again, there was a clear difference between the groups, $t(74)=-6.09, p<.001, d=1.40, B F>1000$, with the high-ADHD group reporting more state regulation difficulties. Furthermore, there was a high correlation between the scores of the prescreening (short ASRS) and the corresponding questions of the post-hoc full ASRS (Pearson's correlation coefficient $r(74)=.914, p<.001, B F>1000$ ). When running separate correlations for both groups, the correlation was $r=.554$ in the high-ADHD group and $r=.415$ in the low-ADHD group. The scores on the full ASRS and the SRD questionnaire correlated positively with each other, $r(74)=.795, p<.001, B F>1000$. The correlations per group were $r=.766$ for the high-ADHD group and $r=.504$ for the low-ADHD group. This indicates that self-reported ADHD symptomatology is positively related to self-reported difficulties with state regulation. See Figure 3 for scatterplots of the correlations mentioned here. The full ASRS had excellent internal consistency (Cronbach's Alpha = .943), while internal consistency was acceptable for the SRD questionnaire (Cronbach's Alpha $=.756$ ).

[INSERT FIGURE 3]

\subsection{Behavioral performance}

Median RTs were analyzed in a mixed ANOVA using group (high vs. low ADHD) as betweensubject factor and ER (fast, moderate, and slow) as within-subject factor. The results are listed in Table 1. In the median RT model, planned contrasts showed that the ER $\times$ Group interaction 
had a linear effect (see Table 1). Both the F-test and the Bayes' Factor (BF) further confirmed the presence of a main effect of ER (BF: decisive evidence), and pairwise comparisons showed significant RT increments from the fast to the moderate ER, $t(74)=13.20, p<.001$, and from the moderate to the slow $E R, t(74)=9.17, p<.001$. Moreover, a main effect of group (BF: substantial evidence) was found, indicating overall longer RT for the high-ADHD group. Figure 4 provides a graphical overview of these effects.

[INSERT FIGURE 4]

In the RT variability model, a planned quadratic contrast revealed the expected quadratic effect in the ER $\times$ Group interaction (see Table 1). The main effect of ER was neither clearly present or absent in our data, as the $F$-test result was marginally significant, accompanied by a $B F$ value indicating anecdotal evidence in favor of the null hypothesis. Neither the F-statistic nor the BF value confirmed a main effect of group, they both favored the null hypothesis. Separate group analyses revealed that the quadratic interaction contrast was driven by a quadratic ER effect in the high-ADHD group, $t(38)=1.99, p=.050$, which showed higher RT variability in extreme ERs. This effect was not present in the low-ADHD group, $t(36)=0.88, p$ $=.385$. Taken together, this indicates that state regulation deficits in the high-ADHD group were not only reflected in the median RT, but also in the RT variability. Since the conditions contained unequal numbers of trials, which may affect the robustness of the findings, the results were co-validated with a bootstrapping method. This approach gave similar results (see Supplementary Materials 2).

[INSERT TABLE 1] 
Error rates were very low: The percentage of omission errors on Go-trials was $0.0 \%-0.2 \%$ in the low-ADHD group and $0.1 \%-0.3 \%$ in the high-ADHD group, and the percentage of commission errors on No-Go-trials was $0.4 \%-1.5 \%$ in the low-ADHD group and $0.5 \%-2.2 \%$ in the high-ADHD group. Statistical differences could not be calculated due to the lack of data points.

\subsection{Phasic pupil size}

We observed a clear dilation of the pupil following target trials, and a smaller constriction following standard trials. Both of these reactions are plotted in Figure 2, exemplarily showing the pupil reactions of fast ER condition. The peak (i.e., maximum amplitude) values were analyzed in a mixed ANOVA, with a final sample of $n=31$ high-ADHD and $n=32$ low-ADHD. There was a significant main effect of $E R, F(2,122)=34.81, p<.001, \eta^{2}{ }_{p}=.363, B F>1000$. Planned contrasts and Bayesian tests confirmed that the ER effect was driven by a significantly greater peak amplitude in the slow ER compared to the moderate ER, $p<.001$ and BFcorrected $>1000$, while there was no significant difference between the moderate and the fast $\mathrm{ER}, p=.526$, BFcorrected $=0.34$. Neither the group main effect nor the interaction $E R \times$ Group were significant $(F(1,61)=1.37, p=.247, B F=0.23$, and $F(2,122)=0.81, p=.446, B F=0.39$, respectively). These results are depicted in Figure 5 (left). To conclude, ER influenced the phasic pupil response such that it was larger in the slow ER compared to both other conditions. However, group differences were not observed.

\subsection{Tonic pupil response}

In order to analyze the impact of ER on pre-stimulus pupil size, we used the average of all 200 ms pre-stimulus segments per participant and ER condition. A mixed ANOVA showed a signif- 
icant main effect of $E R, F(2,122)=9.81, p<.001, \eta^{2} p=.139, B F=142.28$. There was no significant main group effect, $F(1,61)=0.01, p=.944, B F=0.43$, nor was there an interaction effect ER $\times$ Group, $F(2,122)=0.19, p=.828, B F=0.17$. Surprisingly, planned contrasts and Bayesian tests revealed a lower average pre-stimulus baseline in the fast ER compared to the moderate $\mathrm{ER}, t(61)=-4.10, p<.001$, and BFcorrected $=152.29$. Again contrary to our hypothesis, no difference was found between moderate ER and slow ER, $t(61)=-0.61, p=.545$, and BFcorrected $=0.17$. Note that group sizes were $n=32$ for high-ADHD, and $n=31$ for low-ADHD.

The $15 \mathrm{~s}$ baseline intervals at the condition midpoint served as a secondary means of investigating tonic pupil size. A mixed ANOVA showed that there was a clear main effect of ER, $F(2,136)=9.24, p<.001, \eta_{p}^{2}=.120, B F=98.74$. Planned comparisons and Bayesian tests showed a significantly lower pupil size in the fast ER compared to the moderate ER, $t(68)=-$ $3.67, p<.001$, BFcorrected $=84.79$, but equal pupil size in the moderate and slow ER, $t(68)=$ $-0.12, p<.909$, BFcorrected $=0.13$. This is in line with what was found for the pre-stimulus baseline averages. There was neither a main group effect, $F(1,68)=0.24, p=.627, B F=0.34$, nor an interaction effect $E R \times G r o u p, F(2,136)=0.10, p=.907, B F=0.12$. Note that the group sizes were: $n=34$ high-ADHD, $n=36$ low-ADHD. Figure 5 (right) represents the results of the baseline period tonic pupil size. Hence, similar effects were found for the two indices of tonic pupil size: ER had an effect on tonic pupil size, although group differences were absent.

[INSERT FIGURE 5]

\subsection{Correlations: effort allocation and performance}


RT findings clearly showed state regulation difficulties in the high-ADHD group, but not in the low-ADHD group. However, no group differences were found for the amount of cognitive effort allocated (i.e., the phasic pupil response). To gain additional insight, we calculated the correlation between phasic pupil amplitude (slow - fast condition) and median RT (slow - fast condition). A significant negative correlation was found, in spite of an indecisive $B F$ value: $r(65)$ $=-.25, p=.045, B F=1.1$. This suggests that a larger increase in transient pupil amplitude (additional cognitive effort) between the slow and the fast ER could possibly be associated with a smaller increase in RT (smaller performance decline) between the slow and the fast ER. Importantly, analyses per group showed that this association was somewhat more evident for the low-ADHD group, with slightly more decisive significance and $B F$ values, $r(33)=-.38, p=$ $.029, B F=2.12$ ), while no significant relationship between additional effort allocation and performance was observed for the high-ADHD group, $r(32)=-.20, p=.267, B F=0.39)$. However, the difference between the groups' correlations was not significant, Fisher's $Z=-0.78, p=.217$.

\section{Discussion}

In the current experiment, we used performance and pupillometry indices to test the relationship between ADHD symptom levels, behavior, tonic pupil size and phasic pupil reactions. There were two novel elements in this approach. Firstly, the SRD account was tested with three ER conditions and pupil size measurement. Secondly, this was done with non-diagnosed adult participants, with either high or low ADHD symptom levels, based on the widespread notion that ADHD symptomatology should be regarded as a continuous trait (McLennan, 2016). The behavioral results clearly indicate state regulation difficulties in adults with more ADHD symptoms. RT became slower with slower ERs and, importantly, this effect was more pronounced in the high-ADHD group, in line with previous research findings with clinical ADHD 
groups (Metin et al., 2012). Moreover, the ER manipulation affected RT variability especially in the high-ADHD group, with higher RT variability showing an U-curve effect, again in line with previous findings in a clinical ADHD sample (Metin et al., 2014). Besides, adults with more ADHD symptoms also reported more state regulation difficulties, as assessed with the SRD questionnaire. The pupillometry results were surprising: We found a main effect of ER on the amplitude of the phasic pupil responses, resulting from larger amplitudes during slow ERs. ER also impacted tonic pupil size with a significantly lower pupil size in the fast condition. In spite of this, neither pupil measure showed group differences. The results challenge the hypothesis of LC dysfunction being at the core of ADHD-related state regulation difficulties.

\subsection{Behavioral performance}

The two experimental groups had equal sample characteristics, but were selected to differ strongly in terms of ADHD symptoms, which was confirmed by additional questionnaire data obtained during the experiment session. The RT results, which showed an increase with decreasing ER, replicated previous findings (see the meta-analysis by Metin et al., 2012), including these of the preliminary pupillometry study by Metin et al. (2017), who applied the same paradigm with children clinically diagnosed with ADHD. This effect has been argued to be the result of a suboptimal activation state when stimuli are presented at slow pace (van der Meere, Shalev, Borger, \& Wiersema, 2009) Importantly, the SRD account predicts this effect to be more pronounced in individuals with $A D H D$, as they have difficulty with up-regulating a non-optimal activation state. This up-regulation, however, is required to keep performance at a comparable level to control groups. We indeed observed this effect to be more pronounced in the high-ADHD group, which is in line with previous ADHD research findings. (Benikos \& Johnstone, 2009; Metin et al., 2014; van der Meere et al., 1995). Additionally, we found a U- 
shaped RT variability effect for the high-ADHD group, indicating state regulation difficulties at the extreme ERs. Albeit indicative of state regulation deficits, this effect is not consistently found in previous research, which may depend on how variability is quantified. Metin et al. (2014) applied ex-Gaussian modeling and also found an accentuated quadratic relationship between event rate and Gaussian variability in the ADHD group compared to the controls.

Unlike in most previous experiments, accuracy was very high, rendering a statistical comparison impossible. This was expected as we used a target detection task in contrast to most previous studies that used a Go/No-Go task, loading high on inhibitory control and consequently eliciting many more inhibition errors (Metin et al., 2012).

In general, the behavioral findings clearly indicate state regulation difficulties in undiagnosed adults with more ADHD symptoms, in line with previous studies that included individuals with a diagnosis of ADHD (Metin et al., 2017). The finding that adults with more ADHD symptoms reported more state regulation difficulties in daily life, as assessed by the SRD questionnaire, provides further support. It is important to note here that the SRD questionnaire was a newly developed instrument and future research is warranted to further investigate its psychometric qualities.

\subsection{Phasic pupil effects}

Phasic pupil size, an index of effort allocation, was expected to show larger amplitudes in the fast and the slow ER, compared to the medium ER, since we expected that more compensatory effort would be required under hyper- and hypo-stimulating conditions. However, the phasic pupil amplitudes increased with decreasing ER, driven by a significantly higher peak amplitude in the slow ER. Metin et al. (2017) did find the same effect as the phasic results here, but only in the group of children with ADHD, while a U-shaped effect was found in the control group. 
Although speculative, it may be that the fast condition led to a state of over-activation in children, for which the control children compensated with compensatory cognitive effort, while in the current study the fast condition was not stimulating enough to induce a state of overactivation in adults. Apart from the tonic pupil findings, the data do however suggest that the slow condition lead to under-activation, and that additional effort allocation was used to counteract performance decline in the slow condition. Correlational analyses further suggested that the more additional cognitive effort was allocated (increase in phasic pupil response with decreasing ERs), the less the performance decline (smaller increase of RT with decreasing ERs).

Taken together, despite similar levels of additional effort allocation, the cognitive effort invested by adults with more ADHD symptoms does not 'translate' into equal beneficial effects at the performance level. Previous studies on diagnosed individuals with ADHD that included other indices of cognitive effort (e.g., P3 and heart rate variability), concluded that the state regulation deficits as observed in the ADHD group were the result of less effort allocation (Börger et al., 1999; Wiersema, Van der Meere, Antrop, et al., 2006). An interesting avenue for future research would be to investigate whether diagnosed individuals with ADHD indeed differ from undiagnosed individuals with elevated levels of ADHD symptomatology, in terms of the disrupted mechanism underlying their state regulation difficulties.

\subsection{Tonic pupil effects}

Tonic pupil size was analyzed using two conceptually different approaches, in order to examine the validity of the dominant approach to baseline pupil size. On the one hand, the pupil size of the 200 ms pre-stimulus baseline segments was averaged per condition and compared between ERs. On the other hand, the $15 \mathrm{~s}$ baseline periods at the mid-point of each block were compared across ERs. Importantly, we obtained the same general results for both indices of 
tonic pupil size, namely a reduced pupil size in the fast condition compared to the other conditions.

The finding was opposite to our prediction, that was based on the assumption as that tonic pupil size reflects tonic arousal and that tonic arousal resembles the construct of activation in the CEM and SRD accounts. According to the CEM, ER influences the activation level, with lower activation levels for slower ERs. Hence, the largest tonic pupil size was expected in the fast ER, the smallest in the slow condition, and a medium pupil size for the moderate ER condition. The results were surprising, as they ran counter to this hypothesis, with the smallest pupil size in the fast condition. This would signify that the tonic arousal level was lowest in the most stimulating condition, which is quite improbable. A key assumption of the LC theory by Aston-Jones and Cohen (2005) is that tonic activity of the LC is reflected in tonic pupil size, which forms an index of tonic arousal. In the light of the tonic pupil results in the current experiment, it appears that the link between tonic pupil size and activation is far from straightforward, and consequently that tonic pupil size may not be a suitable index of the activation state.

The findings are more in favor of another interpretation derived from research about mind wandering. Mind wandering entails the activation of the Default Mode Network (Christoff et al., 2009). A growing number of studies have shown that tonic pupil size (mostly measured as pre-stimulus pupil size) is larger when participants are mind wandering (Franklin et al., 2013; Unsworth \& Robison, 2016, 2017), which in turn is likely mediated by activation of the Default Mode Network (Fan et al., 2012; Yellin et al., 2015). It is safe to presume that in the current experiment, the slow ER provided more mind wandering opportunity due to the low task de- 
mand. Notwithstanding, the absence of a group difference remains puzzling, as mind wandering is likely to occur more frequently in individuals with ADHD (Seli et al., 2015; Shaw \& Giambra, 1993), which has even led to the 'mind wandering hypothesis of ADHD' (Bozhilova et al., 2018). This calls for further research that includes alternative measures of tonic arousal, such as skin conductance, and assessment of mind wandering.

\subsection{Limitations}

The results of this study are not generalizable without certain limitations. Firstly, the study did not involve a clinical group of patients with ADHD. Even though the ASRS is a validated instrument, participants only reported their subjective ADHD symptoms and no behavioral assessment was carried out. Furthermore, ADHD symptoms can be mimicked by other psychopathologies. Although participants with a diagnosis of a psychiatric or neurological disorder of any kind were excluded from this experiment, we did not systematically gather data on the presence of other (dimensional) psychopathologies. Reliably controlling for their potential influence would have severe implications for power and would require a manifold larger sample size. The current study therefore has the limitation that the influence of other disorders, albeit on subclinical levels, cannot be ruled out. Another caveat is that we did not collect data on IQ or socio-economic status, which may have influenced our findings. However, the influence of these factor is expected to be small as we recruited a relatively homogeneous sample of participants (university students). In light of these limitations, the present results should be interpreted with caution.

\subsection{Conclusion}


In the present findings, the behavioral performance effects as well as the self-reports clearly indicated more state regulation difficulties in undiagnosed adults with more ADHD symptoms, in line with previous ADHD studies that included individuals with a formal diagnosis of ADHD. Despite similar levels of additional effort allocation, as reflected by phasic pupil responses, the cognitive effort invested by adults with more ADHD symptoms does not seem to 'translate' into similar effects at the performance level. Moreover, the findings cast doubt on the validity of tonic pupil size for measuring the activation state. The lack of group differences for both pupil indices appears to question the contribution of the LC-noradrenergic system to state regulation difficulties related to ADHD symptom level. Given the ongoing nature of research and the limits to our current knowledge, the SRD account needs to be tested further and revised as our understanding progresses.

Funding information: This work was supported by the Research Foundation Flanders (FWO), grant number G057218, awarded to JRW.

Acknowledgements: The authors are grateful to Nina Seminck for her help during data acquisition. They would also like thank Henk van Steenbergen for his support.

Author Note: The data of this study have been presented at the Eunethydis Meeting 2019. 


\section{Bibliography}

Adler, L., Spencer, T. J., Faraone, S. V., Kessler, R. C., Howes, M. J., Biederman, J., \& Secnik, K. (2006). Validity of pilot adult ADHD Self-Report Scale (ASRS) to rate adult ADHD symptoms. Annals of Clinical Psychiatry, 18(3), 145-148.

https://doi.org/10.1080/10401230600801077

Alnaes, D., Sneve, M. H., Espeseth, T., Endestad, T., van de Pavert, S. H. P., \& Laeng, B. (2014). Pupil size signals mental effort deployed during multiple object tracking and predicts brain activity in the dorsal attention network and the locus coeruleus. Journal of Vision, 14(4), 1-1. https://doi.org/10.1167/14.4.1

American Psychiatric Association. (2013). Diagnostic and Statistical Manual of Mental Disorders: DSM-5 (5th ed.). Author.

https://doi.org/10.1176/appi.books.9780890425596

Aston-Jones, G., \& Cohen, J. D. (2005). An Integrative Theory of Locus CoeruleusNorepinephrine Function: Adaptive Gain and Optimal Performance. Annual Review of Neuroscience, 28(1), 403-450.

https://doi.org/10.1146/annurev.neuro.28.061604.135709

Benikos, N., \& Johnstone, S. J. (2009). Arousal-state modulation in children with AD/HD. Clinical Neurophysiology, 120(1), 30-40. https://doi.org/10.1016/j.clinph.2008.09.026

Börger, N. A., \& van der Meere, J. J. (2000). Motor control and state regulation in children with ADHD: a cardiac response study. Biological Psychology, 51(2-3), 247-267. https://doi.org/10.1016/S0301-0511(99)00040-X

Börger, N. A., Van der Meere, J. J., Ronner, a, Alberts, E., Geuze, R., \& Bogte, H. (1999). Heart rate variability and sustained attention in ADHD children. Journal of Abnormal Child Psychology, 27(1), 25-33. https://doi.org/10.1023/A:1022610306984

Bozhilova, N., Michelini, G., Kuntsi, J., \& Asherson, P. (2018). Mind wandering perspective on ADHD. Neuroscience and Biobehavioral Reviews, 92(July), 464-476. https://doi.org/10.1016/j.neubiorev.2018.07.010

Broyd, S. J., Helps, S. K., \& Sonuga-Barke, E. J. S. (2011). Attention-induced deactivations in very low frequency EEG oscillations: Differential localisation according to ADHD symptom status. PLOS ONE, 6(3), 1-8. https://doi.org/10.1371/journal.pone.0017325

Christoff, K., Gordon, A. M., Smallwood, J. M., Smith, R., \& Schooler, J. W. (2009). Experience sampling during $\mathrm{fMRI}$ reveals default network and executive system contributions to mind wandering. Proceedings of the National Academy of Sciences of the United States of America, 106(21), 8719-8724. https://doi.org/10.1073/pnas.0900234106

Crosbie, J., Arnold, P., Paterson, A., Swanson, J., Dupuis, A., Li, X., Shan, J., Goodale, T., Tam, C., Strug, L. J., \& Schachar, R. J. (2013). Response inhibition and ADHD traits: Correlates and heritability in a community sample. Journal of Abnormal Child Psychology, 41(3), 497-507. https://doi.org/10.1007/s10802-012-9693-9

Fan, J., Xu, P., van Dam, N. T., Eilam-Stock, T., Gu, X., Luo, Y. jia, \& Hof, P. R. (2012). Spontaneous brain activity relates to autonomic arousal. Journal of Neuroscience, 
32(33), 11176-11186. https://doi.org/10.1523/JNEUROSCI.1172-12.2012

FH Johanneum. (2018). Presentation Extension for Tobii Pro. User's Manual (Version 3.0.2). FH Johanneum Gesellschaft mbH. https://idk.fh-joanneum.at/wpcontent/uploads/2018/04/tobiipro_et_extension.pdf

Finke, K., Schwarzkopf, W., Müller, U., Frodl, T., Müller, H. J., Schneider, W. X., Engel, R. R., Riedel, M., Möller, H., \& Hennig-Fast, K. (2011). Disentangling the adult attention-deficit hyperactivity disorder endophenotype: Parametric measurement of attention. Journal of Abnormal Psychology, 120(4), 890-901. https://doi.org/10.1037/a0024944

Franklin, M. S., Broadway, J. M., Mrazek, M. D., Smallwood, J. M., \& Schooler, J. W. (2013). Window to the wandering mind: Pupillometry of spontaneous thought while reading. Quarterly Journal of Experimental Psychology, 66(12), 2289-2294. https://doi.org/10.1080/17470218.2013.858170

Garnier-Dykstra, L. M., Pinchevsky, G. M., Caldeira, K. M., Vincent, K. B., \& Arria, A. M. (2010). Self-reported Adult Attention-Deficit/Hyperactivity Disorder Symptoms Among College Students. Journal of American College Health, 59(2), 133-136. https://doi.org/10.1080/07448481.2010.483718

Hong, L., Walz, J. M., \& Sajda, P. (2014). Your eyes give you away: Prestimulus changes in pupil diameter correlate with poststimulus task-related EEG dynamics. PLoS ONE, 9(3). https://doi.org/10.1371/journal.pone.0091321

Howells, F. M., Stein, D. J., \& Russell, V. A. (2012). Synergistic tonic and phasic activity of the locus coeruleus norepinephrine (LC-NE) arousal system is required for optimal attentional performance. Metabolic Brain Disease, 27(3), 267-274. https://doi.org/10.1007/s11011-012-9287-9

Hudziak, J. J., Achenbach, T. M., Althoff, R. R., \& Pine, D. S. (2007). A dimensional approach to developmental psychopathology. International Journal of Methods in Psychiatric Research, 16(S1), S16-S23. https://doi.org/10.1002/mpr.217

Imeraj, L., Sonuga-Barke, E. J. S., Antrop, I., Roeyers, H., Wiersema, J. R., Bal, S., \& Deboutte, D. (2012). Altered circadian profiles in attention-deficit/hyperactivity disorder: An integrative review and theoretical framework for future studies. Neuroscience \& Biobehavioral Reviews, 36(8), 1897-1919. https://doi.org/10.1016/j.neubiorev.2012.04.007

JASP Team. (2020). JASP (Version 0.13.1). https://jasp-stats.org/

Johnson, K. A., Wiersema, J. R., \& Kuntsi, J. (2009). What would Karl Popper say? Are current psychological theories of ADHD falsifiable? Behavioral and Brain Functions, 5(1), 15. https://doi.org/10.1186/1744-9081-5-15

Joshi, S., Li, Y., Kalwani, R. M., \& Gold, J. I. (2016). Relationships between Pupil Diameter and Neuronal Activity in the Locus Coeruleus, Colliculi, and Cingulate Cortex. Neuron, 89(1), 221-234. https://doi.org/10.1016/j.neuron.2015.11.028

Kahneman, D. (1973). Attention and Effort. Prentice-Hall Inc. https://www.jstor.org/stable/1421603?origin=crossref 
Kessler, R. C., Adler, L. A., Gruber, M. J., Sarawate, C. A., Spencer, T. J., \& Van Brunt, D. L. (2007). Validity of the World Health Organization Adult ADHD Self-Report Scale (ASRS) Screener in a representative sample of health plan members. International Journal of Methods in Psychiatric Research, 16(2), 52-65. https://doi.org/10.1002/mpr.208

Kessler, R. C., Adler, L., Ames, M., Demler, O., Faraone, S., Hiripi, E., Howes, M. J., Jin, R., Secnik, K., Spencer, T. J., Ustun, T. B., \& Walters, E. E. (2005). The World Health Organization adult ADHD self-report scale (ASRS): A short screening scale for use in the general population. Psychological Medicine, 35(2), 245-256. https://doi.org/10.1017/S0033291704002892

Konica Minolta. (2013). Luminance Meter LS-100, LS-110 Instruction Manual. https://www.konicaminolta.com/instruments/download/instruction_manual/light/pdf/ Is-100-110_instruction_eng.pdf

Konrad, K., Neufang, S., Hanisch, C., Fink, G. R., \& Herpertz-Dahlmann, B. (2006). Dysfunctional Attentional Networks in Children with Attention Deficit/Hyperactivity Disorder: Evidence from an Event-Related Functional Magnetic Resonance Imaging Study. Biological Psychiatry, 59(7), 643-651. https://doi.org/10.1016/j.biopsych.2005.08.013

Mathôt, S. (2018). Pupillometry: Psychology, Physiology, and Function. Journal of Cognition, 1(1), 1-23. https://doi.org/10.5334/joc.18

McLennan, J. D. (2016). Understanding attention deficit hyperactivity disorder as a continuum. Canadian Family Physician, 62(12), 979-982. https://doi.org/10.1007/SpringerReference_34414

Metin, B., Krebs, R. M., Wiersema, J. R., Verguts, T., Gasthuys, R., van der Meere, J. J., Achten, E., Roeyers, H., \& Sonuga-Barke, E. J. S. (2015). Dysfunctional modulation of default mode network activity in attention-deficit/hyperactivity disorder. Journal of Abnormal Psychology, 124(1), 208-214. https://doi.org/10.1037/abn0000013

Metin, B., Krebs, R. M., Wiersema, J. R., Verguts, T., Gasthuys, R., Van der Meere, J. J., Achten, E., Roeyers, H., \& Sonuga-Barke, E. J. S. (2015). Dysfunctional modulation of default mode network activity in attention-deficit/hyperactivity disorder. Journal of Abnormal Psychology, 124(1), 208-214. https://doi.org/10.1037/abn0000013

Metin, B., Roeyers, H., Wiersema, J. R., van der Meere, J. J., \& Sonuga-Barke, E. J. S. (2012). A meta-analytic study of event rate effects on Go/No-Go performance in attentiondeficit/hyperactivity disorder. Biological Psychiatry, 72(12), 990-996. https://doi.org/10.1016/j.biopsych.2012.08.023

Metin, B., Sonuga-Barke, E. J. S., Wiersema, J. R., Roeyers, H., \& Vermeir, S. (2017). The differential effect of event rate on pupil dilation patterns suggests effort dysregulation problems in ADHD [Abstract]. European Psychiatry, 41, 5635. https://doi.org/10.1016/j.eurpsy.2017.01.1042

Metin, B., Wiersema, J. R., Verguts, T., Gasthuys, R., van Der Meere, J. J., Roeyers, H., \& Sonuga-Barke, E. J. S. (2014). Event rate and reaction time performance in ADHD: Testing predictions from the state regulation deficit hypothesis using an ex-Gaussian model. Child Neuropsychology, 22(1), 99-109. 
https://doi.org/10.1080/09297049.2014.986082

Mohamed, S. M. H., Börger, N. A., Geuze, R. H., \& Van der Meere, J. J. (2016). Post-error adjustments and ADHD symptoms in adults: The effect of laterality and state regulation. Brain and Cognition, 108, 11-19. https://doi.org/10.1016/j.bandc.2016.06.006

Murphy, P. R., Robertson, I. H., Balsters, J. H., \& O'Connell, R. G. (2011). Pupillometry and P3 index the locus coeruleus-noradrenergic arousal function in humans. Psychophysiology, 48(11), 1532-1543. https://doi.org/10.1111/j.1469-8986.2011.01226.x

Nilsen, E. S., Mewhort Buist, T. A., Gillis, R., \& Fugelsang, J. (2013). Communicative Perspective-Taking Performance of Adults With ADHD Symptoms. Journal of Attention Disorders, 17(7), 589-597. https://doi.org/10.1177/1087054711428947

Panagiotidi, M., Overton, P., \& Stafford, T. (2017). Increased microsaccade rate in individuals with ADHD traits. Journal of Eye Movement Research, 10(1), 1-9. https://doi.org/10.16910/10.1.6

Peavler, W. S. (1974). Pupil Size, Information Overload, and Performance Differences. Psychophysiology, 11(5), 559-566. https://doi.org/10.1111/j.1469-8986.1974.tb01114.x

Pribram, K. H., \& McGuinness, D. (1975). Arousal, activation, and effort in the control of attention. Psychological Review, 82(2), 116-149. https://doi.org/10.1037/h0076780

Rajkowski, J., Kubiak, P., \& Aston-Jones, G. (1994). Locus coeruleus activity in monkey: Phasic and tonic changes are associated with altered vigilance. Brain Research Bulletin, 35(56), 607-616. https://doi.org/10.1016/0361-9230(94)90175-9

Reimer, J., McGinley, M. J., Liu, Y., Rodenkirch, C., Wang, Q., McCormick, D. A., \& Tolias, A. S. (2016). Pupil fluctuations track rapid changes in adrenergic and cholinergic activity in cortex. Nature Communications, 7(May), 1-7. https://doi.org/10.1038/ncomms13289

Sanders, A. F. (1983). Towards a model of stress and human performance. Acta Psychologica, 53(1), 61-97. https://doi.org/10.1016/0001-6918(83)90016-1

Scheres, A., Oosterlaan, J., \& Sergeant, J. A. (2001). Response Execution and Inhibition in Children with AD/HD and Other Disruptive Disorders: The Role of Behavioural Activation. Journal of Child Psychology and Psychiatry, 42(3), S0021963001006898. https://doi.org/10.1017/S0021963001006898

Schneider, M., Hathway, P., Leuchs, L., Sämann, P. G., Czisch, M., \& Spoormaker, V. I. (2016). Spontaneous pupil dilations during the resting state are associated with activation of the salience network. Neurolmage, 139, 189-201. https://doi.org/10.1016/j.neuroimage.2016.06.011

Seli, P., Smallwood, J. M., Cheyne, J. A., \& Smilek, D. (2015). On the relation of mind wandering and ADHD symptomatology. Psychonomic Bulletin \& Review, 22(3), 629-636. https://doi.org/10.3758/s13423-014-0793-0

Sergeant, J. A. (2000). The cognitive-energetic model: an empirical approach to AttentionDeficit Hyperactivity Disorder. Neuroscience \& Biobehavioral Reviews, 24(1), 7-12. https://doi.org/10.1016/S0149-7634(99)00060-3

Sergeant, J. A., \& Van der Meere, J. J. (1990). Additive factor method applied to 
psychopathology with special reference to childhood hyperactivity. Acta Psychologica, 74(2-3), 277-295. https://doi.org/10.1016/0001-6918(90)90009-5

Shaw, G. A., \& Giambra, L. (1993). Task-unrelated thoughts of college students diagnosed as hyperactive in childhood. Developmental Neuropsychology, 9(1), 17-30. https://doi.org/10.1080/87565649309540541

Sirois, S., \& Brisson, J. (2014). Pupillometry. Wiley Interdisciplinary Reviews: Cognitive Science, 5(6), 679-692. https://doi.org/10.1002/wcs.1323

Sonuga-Barke, E. J. S., Wiersema, J. R., van der Meere, J. J., \& Roeyers, H. (2010). Contextdependent Dynamic Processes in Attention Deficit/Hyperactivity Disorder: Differentiating Common and Unique Effects of State Regulation Deficits and Delay Aversion. Neuropsychology Review, 20(1), 86-102. https://doi.org/10.1007/s11065009-9115-0

Tobii AB. (2016). Tobii Studio User's Manual (Version 3.4.5). Tobii AB. https://www.tobiipro.com/siteassets/tobii-pro/user-manuals/tobii-pro-studio-usermanual.pdf

Tobii Technology AB. (2014). Tobii TX300 Eye Tracker. User Manual 2nd ed. Tobii Technology AB. https://www.tobiipro.com/siteassets/tobii-pro/user-manuals/tobii-pro-tx300-eyetracker-user-manual.pdf/?v=2.0

Unsworth, N., \& Robison, M. K. (2016). Pupillary correlates of lapses of sustained attention. Cognitive, Affective and Behavioral Neuroscience, 16(4), 601-615. https://doi.org/10.3758/s13415-016-0417-4

Unsworth, N., \& Robison, M. K. (2017). The importance of arousal for variation in working memory capacity and attention control: A latent variable pupillometry study. Journal of Experimental Psychology: Learning Memory and Cognition, 43(12), 1962-1987. https://doi.org/10.1037/xlm0000421

Unsworth, N., \& Robison, M. K. (2018). Tracking arousal state and mind wandering with pupillometry. Cognitive, Affective and Behavioral Neuroscience, 18(4), 638-664. https://doi.org/10.3758/s13415-018-0594-4

van der Meere, J. J. (2005). State Regulation and Attention Deficit Hyperactivity Disorder. In D. Gozal \& D. L. Molfese (Eds.), Attention Deficit Hyperactivity Disorder (pp. 413-433). Humana Press Inc. https://doi.org/10.1385/1-59259-891-9:413

van der Meere, J. J., Shalev, R. S., Börger, N. A., \& Wiersema, J. R. (2009). Methylphenidate, interstimulus interval, and reaction time performance of children with attention deficit/hyperactivity disorder: A pilot study. Child Neuropsychology, 15(6), 554-566. https://doi.org/10.1080/09297040902758803

van der Meere, J. J., Stemerdink, N., \& Gunning, B. (1995). Effects of Presentation Rate of Stimuli on Response Inhibition in ADHD Children with and without Tics. Perceptual and Motor Skills, 81(1), 259-262. https://doi.org/10.2466/pms.1995.81.1.259

van der Wel, P., \& van Steenbergen, H. (2018). Pupil dilation as an index of effort in cognitive control tasks: A review. Psychonomic Bulletin \& Review, 25(6), 2005-2015. https://doi.org/10.3758/s13423-018-1432-y 
van Steenbergen, H. (2008). Pupil analysis toolbox for Brain Vision.

http://www.henkvansteenbergen.com/resources/

van Steenbergen, H., \& Guido, P. H. B. (2013). Pupil dilation in the Simon task as a marker of conflict processing. Frontiers in Human Neuroscience, 7(May), 1-11.

https://doi.org/10.3389/fnhum.2013.00215

Wang, C.-A., Baird, T., Huang, J., Coutinho, J. D., Brien, D. C., \& Munoz, D. P. (2018). Arousal Effects on Pupil Size, Heart Rate, and Skin Conductance in an Emotional Face Task. Frontiers in Neurology, 9(December), 1-13. https://doi.org/10.3389/fneur.2018.01029

Wiegand, I., Hennig-Fast, K., Kilian, B., Müller, H. J., Töllner, T., Möller, H., Engel, R. R., \& Finke, K. (2016). EEG correlates of visual short-term memory as neuro-cognitive endophenotypes of ADHD. Neuropsychologia, 85, 91-99. https://doi.org/10.1016/j.neuropsychologia.2016.03.011

Wiersema, J. R., Van der Meere, J. J., Antrop, I., \& Roeyers, H. (2006). State Regulation in Adult ADHD: An Event-related Potential Study. Journal of Clinical and Experimental Neuropsychology, 28(7), 1113-1126. https://doi.org/10.1080/13803390500212896

Wiersema, J. R., Van der Meere, J. J., Roeyers, H., Van Coster, R., \& Baeyens, D. (2006). Event rate and event-related potentials in ADHD. Journal of Child Psychology and Psychiatry and Allied Disciplines, 47(6), 560-567. https://doi.org/10.1111/j.14697610.2005.01592.x

Willcutt, E. G. (2012). The Prevalence of DSM-IV Attention-Deficit/Hyperactivity Disorder: A Meta-Analytic Review. Neurotherapeutics, 9(3), 490-499. https://doi.org/10.1007/s13311-012-0135-8

Winton, W. M. (1987). Do introductory textbooks present the Yerkes-Dodson Law correctly? American Psychologist, 42(2), 202-203. https://doi.org/10.1037/0003-066X.42.2.202

Yellin, D., Berkovich-Ohana, A., \& Malach, R. (2015). Coupling between pupil fluctuations and resting-state $\mathrm{fMRI}$ uncovers a slow build-up of antagonistic responses in the human cortex. Neurolmage, 106, 414-427. https://doi.org/10.1016/j.neuroimage.2014.11.034

Yerkes, R. M., \& Dodson, J. D. (1908). The relation of strength of stimulus to rapidity of habitformation. Journal of Comparative Neurology and Psychology, 18(5), 459-482. https://doi.org/10.1002/cne.920180503

Zénon, A. (2017). Time-domain analysis for extracting fast-paced pupil responses. Scientific Reports, 7(July 2016), 1-10. https://doi.org/10.1038/srep41484 
Table 1.

Mixed ANOVA results for median Reaction Time (RT) and RT variability

\begin{tabular}{llll}
\hline & & Median RT & RT variability $^{\mathrm{b}}$ \\
\hline \multirow{3}{*}{$\begin{array}{l}\text { Condition (Event } \\
\text { Rate) }\end{array}$} & F-ratio & $F(1.8,136.5)=253.02$ & $F(2,148)=2.74$ \\
& Significance & $p<.001^{* *}$ & $p=.068$ \\
& Effect size & $\eta^{2}{ }_{p}=.774$ & $\eta^{2}=.036$ \\
& Bayes' Factor & $B F>1000$ & $B F=0.41$ \\
\hline \multirow{2}{*}{ Group } & F-ratio & $F(1,74)=6.51$ & $F(1,74)=0.75$ \\
& Significance & $p=.013^{*}$ & $p=.388$ \\
& Effect size & $\eta^{2}{ }_{p}=.081$ & $\eta^{2}=.010$ \\
Polynomial contrasts & Bayes' Factor & $B F=5.25$ & $B F=0.21$ \\
Condition $\times$ Group & Type & Linear & Quadratic \\
& Significance & $F(1,74)=5.23$ & $F(1,74)=4.50$ \\
& Effect size & $\eta^{2}{ }_{p}=.025^{*}$ & $p=.036$ \\
\hline
\end{tabular}

Note. The Bayes' Factor (BF) values show the relative likelihood of the effect, compared to the null effect. ${ }^{\text {aThe }}$ $F$-test for Condition was adjusted for a lack of sphericity using the Huynh-Feldt correction. ${ }^{\mathrm{b}} \mathrm{RT}$ variability was calculated as the quartile-based variability coefficient: Inter-Quartile Range / Median.

${ }^{* *} p<.001 .{ }^{*} p<.05$. 


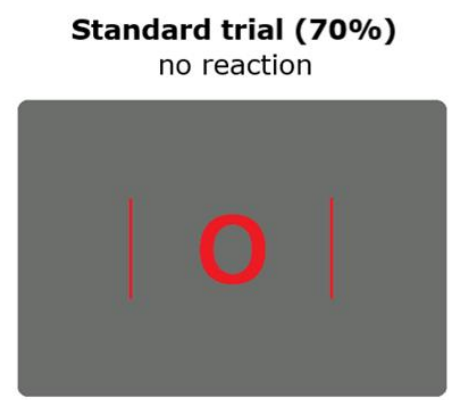

$$
\text { Target trial (30\%) }
$$

press the spacebar

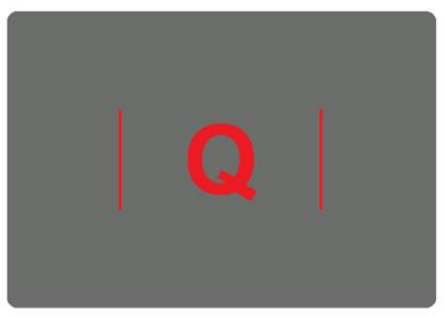

Figure 1. The target detection paradigm used in the present study. Note that the colors are not an accurate representation of the on-screen colors used to achieve equiluminance. The stimuli appeared for 300 ms between the vertical bars, followed by an inter-stimulus interval, wherein only the bars were visible. 

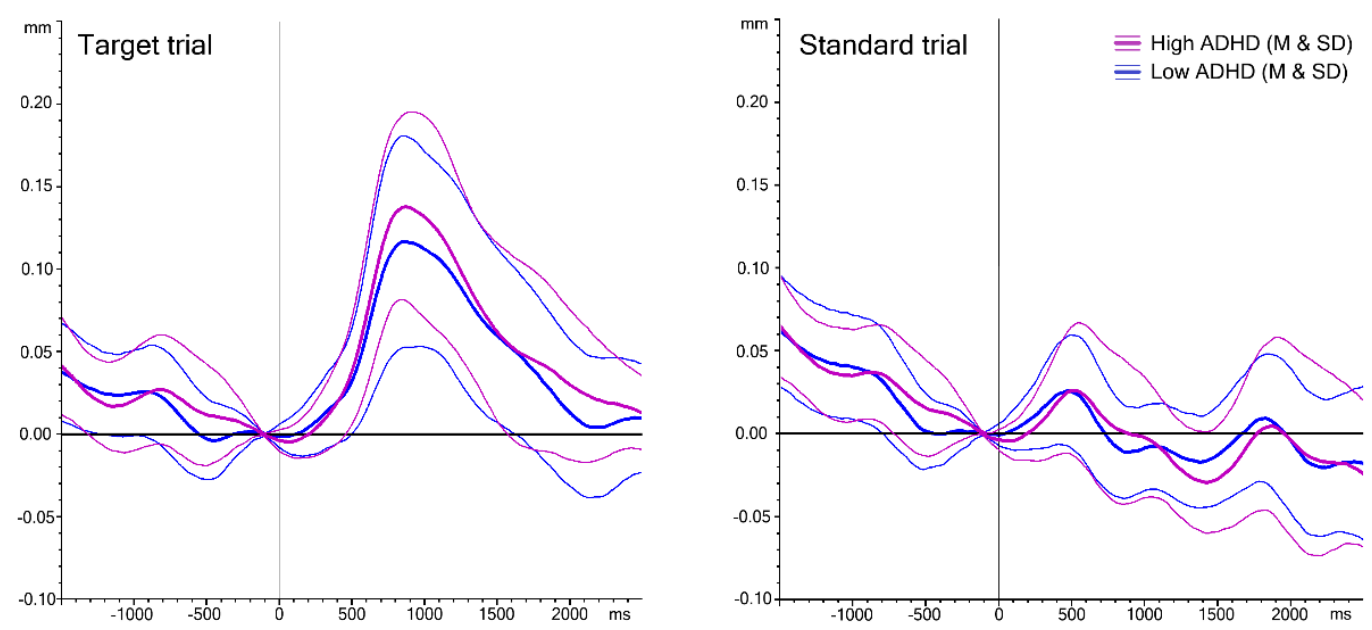

Figure 2. Average signal of the phasic pupil reaction to target trials (left) and standard trials (right), in the fast ER condition. The blue (black) lines are the average and SD of the low-ADHD group, the purple (gray) lines are the average and SD of the high-ADHD group. Note that in both graphs, the trial of interest is preceded and followed by a standard trial. The signal is baseline-corrected using the $200 \mathrm{~ms}$ pre-stimulus interval (-200 to $0 \mathrm{~ms}$ ). 

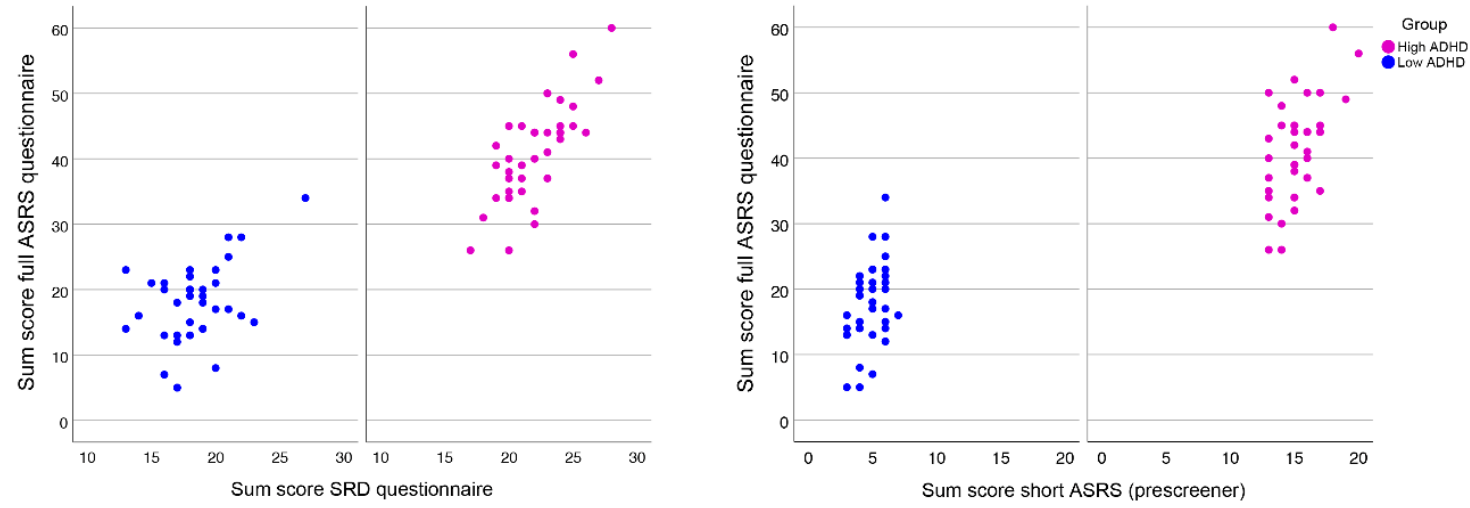

Figure 3. Scatterplots of the correlations of the SRD questionnaire vs. the full ASRS sum score (right) and the sum scores of the short ASRS prescreener vs. the full ASRS (left). 

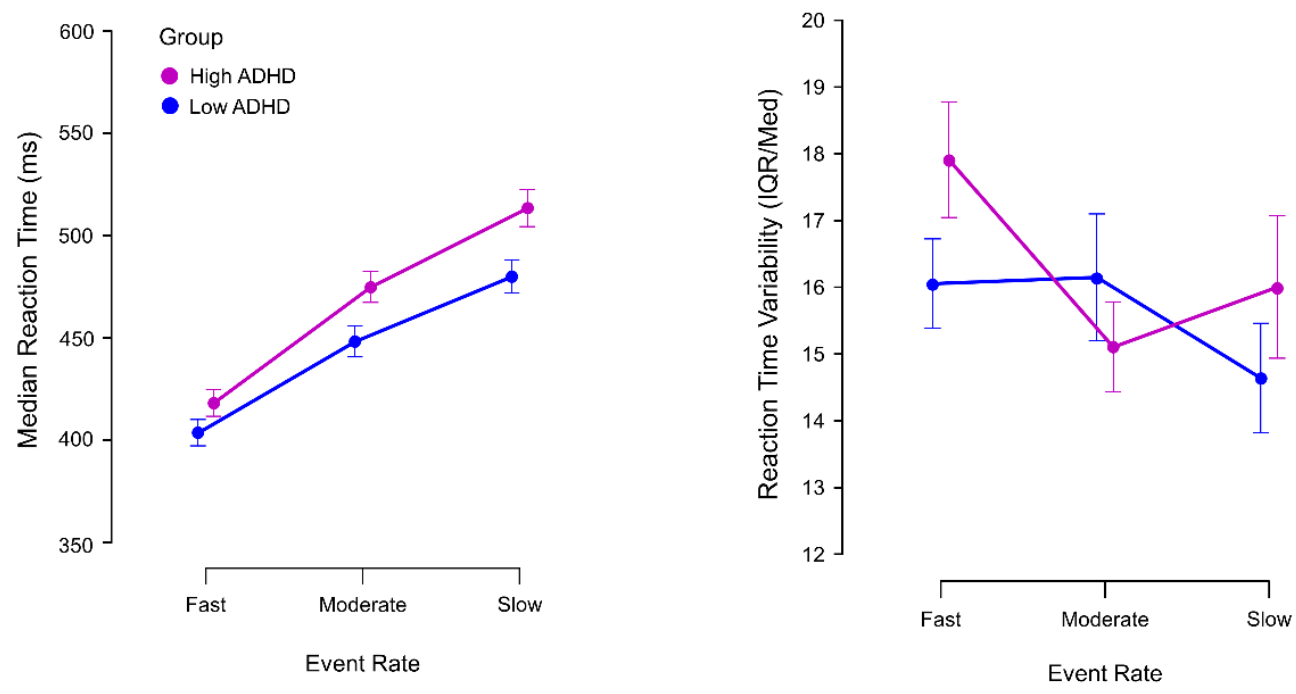

Figure 4. Median reaction time (RT) and RT variability across event rate (ER) conditions. Error bars represent \pm 1 Standard Error around the mean. 

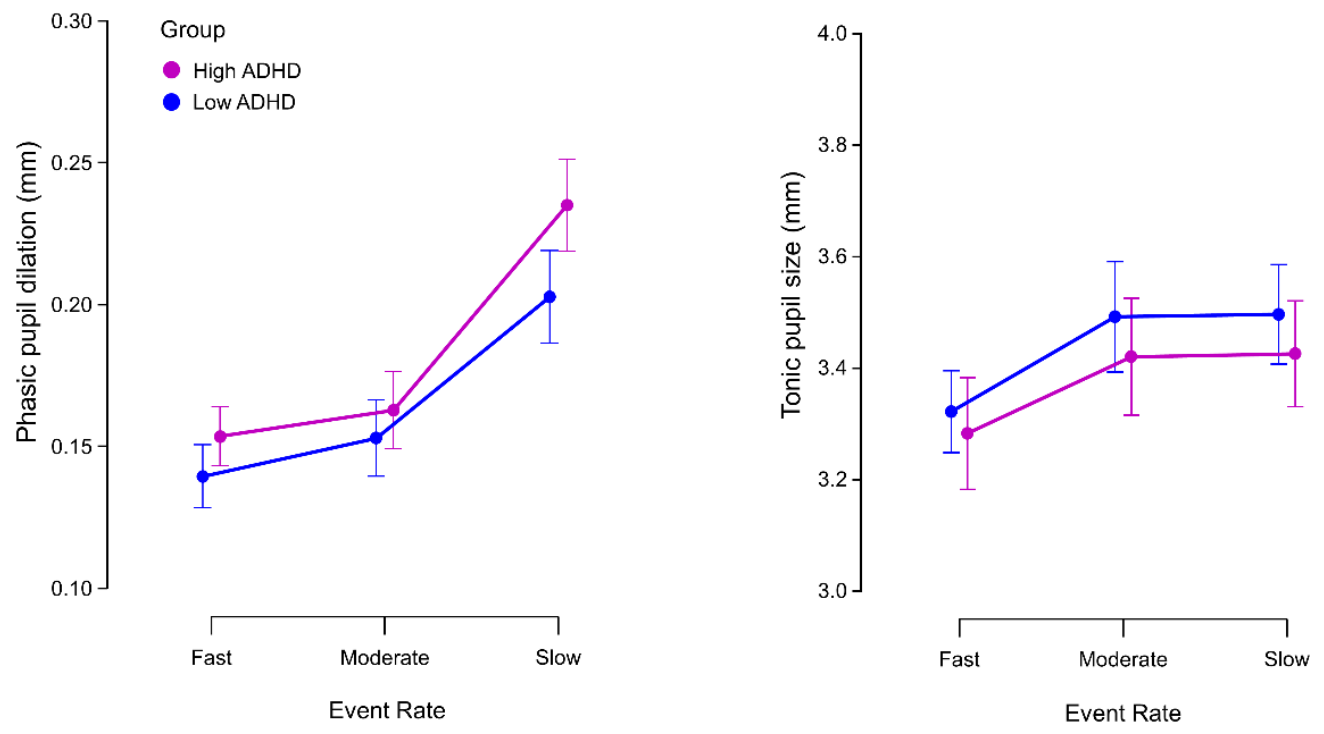

Figure 5. Left: Average amplitude of the phasic pupil reaction to target trials, from baseline to peak, for the different event rate (ER) conditions. Right: Average pupil size of the $15 \mathrm{~s}$ mid-condition baseline period, for the different ER conditions. Error bars represent \pm 1 Standard Error around the mean. 